The Journal of Engineering Research Vol. 5, No.1 (2008) 62-70

\title{
Identification of Friction/Vibration Interaction between Solids
}

\author{
J. A. Abdo*1 and N. Al-Rawahi \\ *1 Department of Mechanical \& Industrial Engineering, College of Engineering, Sultan Qaboos University, P.O. Box 33, \\ Al-khoud 123, Oman
}

Received 24 December 2006; accepted 1 April 2007

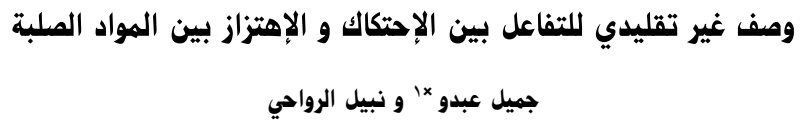

الفلامس: وجد ان قوى الا حتكاك الجاف لا تعتصد فقط على خصائص الاسطح المتلامسة ولكن ايضا على التفاعل الديناميكي بين الاجسام المتلامسة. طور نهوذج رياضي يحتوي على تاثير لزوجة وليونة الاسطح المتلاصسة بحيث ياخذ في عين الاعتبار تفاعل الاسطح الخشنة في دستويات الابعاد البجهرية. النهوذج الرياضي يربط الاهتزاز التفاعلي بين العوامل المتعلقة بعلم تفاعل الاسطح المتحركة نسبيا - ترايبولوجي- (الاحتكاك و التاكل و التزييت) في مستويات الابعاد المجهرية والابعاد الدقيقة' لكتلة موضوعة على حزام متحرك'. خصائص اللزوجة والليونة مستعهله هنا عن طريق إدماج معامل الفقد ومعامل 'ينج' (Young's modulus) للحمول على معامل اشتقاق للتداخل باستخدام نموذج كلفن - فايجوت ( Kelvin-Voigt ). تحليل النظام يؤسس العلاقة بين قوة الاحتكاك والسرعة ويدعم السلوك الملاحظ لعدة أنظمة مع الاحتكاك ـ تطوير النموذج لا يعتد على الطريقة التقليدية للتعامل مع قوة الاحتكاك كنتيجة للتفاعل بين الاسطح الذشنة.عوضا عن ذلك فانة يتم التعامل مع قوة الاحتكاك و السرعة ويدعم السلوك الملاحظ لعدة انظمة مع الاحتكاك وقد نتج عن هذا مجموعة هن البعادلات التفاضلية العادية اللاخطية والتي تربط بشكل مباشر بين اهتزاز النظام وخصائص الاسطح. تم إظهار انه نتيجة للاقتران بين التلاصس و الديناميكية في مستوى الابعاد الدقيقة للنظام، فانة توجد مجموعة من الخصائص في مستويات الابعاد المجهرية و الدقيقة, التي ينتج عنها ميل سالب في العلاقة بين قوة الاحتكاك وسرعة الانزلاق، وهو مصدر معروف لعدم الاستقرار الديناميكي. الهزداه الهنتاهية: التفاعل بين الاحتكاك و الاهتزاز - التفاعل الديناميكي بين الاجسام المتلامسة.

\begin{abstract}
Dry-friction forces have been shown to depend not only on the characteristics of the surface in contact but also on the dynamic interaction of the contacting bodies. A viscoelastic mathematical model that accounts for the interaction at micro-scale of rough surfaces is developed. The mathematical formulation relates the tribological events at microscopic and macroscopic scales vibration response of a "mass on moving belt". The viscoelastic properties are presented by combining loss modulus with Young's modulus to obtain a differential operator on the interference, reminiscent of the Kelvin-Voigt model. The analysis of the system establishes the relation between friction force and speed and supports observed behavior of many systems with friction. The derivations do not rely on a phenomenological account of friction, which requires a presumed friction coefficient. Instead the friction force is accounted for as a result of interaction of the rough surfaces. This has led to a set of nonlinear ordinary differential equations that directly relate the vibration of the system to the surface parameters. It is shown that, as a result of coupling of the macrosystem's dynamics and contact, there are combinations of parameters at micro- and macroscale that yield negative slope in friction force/sliding speed relation, a well known source of dynamic instability.
\end{abstract}

Keywords: Friction/vibration interaction, Dynamic interaction of surfaces

\section{Introduction}

Contact phenomena involving deformable bodies thrive in industry and everyday life. Many authors have considered the mechanical system vibration due to surface interaction and examine the surface theories with the presence of friction (Brockley, et al. 1970; Soom and Kim, 1983; Ibrahim, and Rivin, 1994; Ibrahim, 1994; Aronov et al.

Corresponding author’s e-mail: jdabdo@squ.edu.om 1984a; Aronov, et al. 1984b; Aronov, et al. 1984c; Tan and Roger, 1998; Tworzydlo, et al. 1992; Tzou, et al. 1998). In contrast to the earlier published research, the classical notion of friction coefficient is entirely abandoned in this work. Instead, friction is obtained as a result of system's dynamic response that includes time-dependent forces at the contact.

Numerous works have been devoted to study the friction-induced vibration. For ease of setup and interpretation an idealized physical system consisting of a mass sliding on a moving belt has been considered very often 
(Panovko, et al. 1965; Nayfeh and Mook, 1979; Mitropolskii and Nguyen, 1997; Popp, 1991; Tondl, 1991; McMillan, 1997 and Thomsen and Fidlin, 2002), and it will be in this present study. The physical contact model and governing equations with the inclusion of the viscoelastic properties are presented in this paper to study the dynamic effects of the surface irregularities as a result of mechanical interactions.

Several analytical or computational methods have been applied to solve the dry friction problems to increase the understanding of friction and vibration phenomenon in mechanical systems. Some of the elastic, elastic-plastic, and plastic contact models are reformulated to estimate a normal and tangential contact force, contact area and contact stiffness. The elastic and the elastic-plastic models developed by Abdo and Farhang (2005) and by Abdo and Shamseldin (2005) in addition to the research results pertinent to the estimation of contact stiffness, Abdo (2006) and Abdo (2005) are used in this study along with the addition of the viscoelastic effects of contact. In this work, the viscoelastic properties are presented by combining loss modulus with Young's modulus to obtain a differential operator on the interference, reminiscent of the Kelvin-Voigt model. There is no assumption of friction/velocity relation made in the formulation of the governing differential equations. The elastic and ratedependent contact force components between rough surfaces are presented to obtain the equations governing the vibration response of mass-spring-damper system.

In this section, we are interested in developing a mathematical formulation relating the tribological events at microscopic and the macroscopic scales vibration response of a mass on a moving belt. An example of the physical system is shown in Fig. 1 which consists of a mass $m$ on a belt that moves at constant speed, $\dot{\mathrm{y}}$. The mass is a rigid body, at time $t$ positioned at $x(t)$ in a fixed frame of coordinates. It is subjected to a normal applied static load $F_{n a}$, linear spring-loading force $K x$, plus damping force $C d x / d t$. The $h_{0}$ indicates the ini tial mean plane separation of the two contacting rough surfaces corresponding to the normal applied static load, $F_{n a}$. The model doesn't assume a friction coefficient; therefore, friction is instead obtained as a result of system's dynamic response that includes time-dependent forces at the contact. The tangential -to-normal contact load ratio, the classical definition of the friction coefficient, is shown to depend on system response that in turn is dependent on both the structural and contact characteristics.

\section{The Dynamic Model}

The basic assumptions of the elastic model along with the shoulder-to-shoulder contact model (Abdo and Farhang 2005), and Abdo 2005 and (Abdo and Shamseldin, 2005) are used in this study to account for the elastic contribution of rough surfaces. Elastic contact model has viewed the surface irregularities as identical asperities with spherical summits differing only in their heights that can be measured from the mean plane separation of two rough surfaces. The misaligned asperities permit shoulder-to-shoulder contact. When two solid bodies are brought into contact, the real contact area will only be a fraction of the apparent macroscopic contact area. The introduction of contact slope allowed the consideration of normal and tangential components of the force.

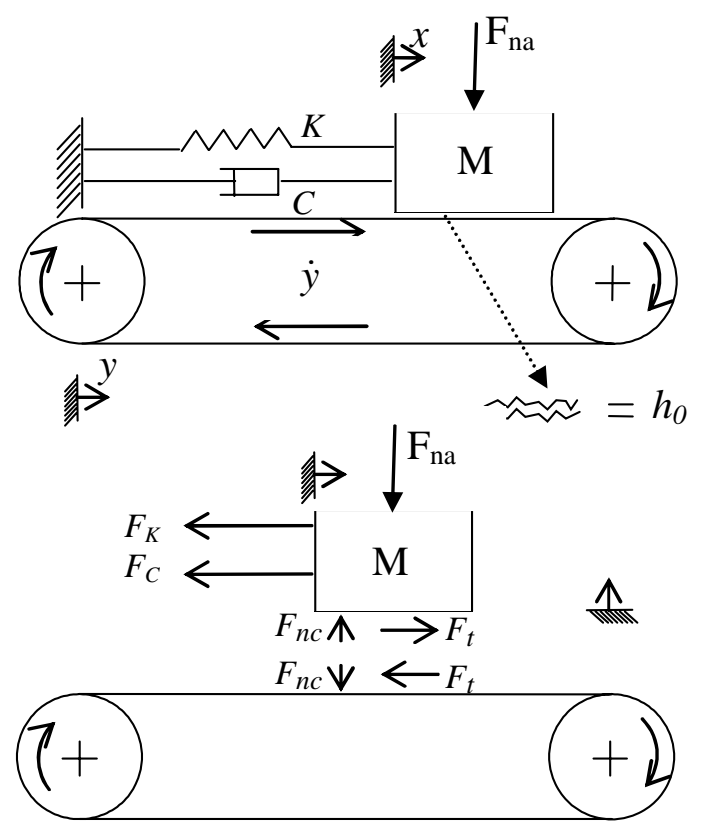

Figure 1. Schematics of block on a moving platform and its free-body diagram $h_{o}$

Referring to the free body diagram shown in Fig. (1), the governing equation of the normal motion can be expressed as

$$
m \ddot{h}-F_{n c}=-F_{n a}
$$

The governing equation of the tangential motion can be expressed as

$$
m \ddot{x}+K x+C \dot{x}=F_{t}
$$

no where $\mathrm{F}_{\mathrm{na}}$ is the normal force applied to mass $M . \mathrm{F}_{\mathrm{nc}}$ is the normal contact force between the two contacting rough surfaces. It is the scalar sum of the resisting and assisting viscoelastic contact components

$$
F_{n c}=F_{n e}^{-}+F_{n e}^{+}+F_{n v}^{-}+F_{n v}^{+}
$$

The terms $F_{n e}^{-}$and $F_{n e}^{+}$denote, respectively, the resisting and assisting elastic normal contacts at the asperity interference slopes (Bengisu et al. 1997; Bengisu et al. 1999), as shown in Fig. 2. It remains a challenge to determine whether there is a net elastic influence on slidi ng surfaces. As shown in Fig. 2 , when in static equilibrium, without the presence of an applied tangential force, the contact force due to negative 
contact slope will be equal to that due to the positive slope; therefore, the net tangential force on a surface is zero. In the presence of an applied tangential force, the equilibrium condition dictates that the net tangential force to be the equilibrating force. Bengisu and Akay (Bengisu et al. 1997) for example assume that the net elastic tangential force, ie. those due to positive slopes minus those due to negative slopes, is negligible. We have chosen instead to include the factor of the friction due to the positive or resisting slopes. In the presence of an applied tangential load that is within the bounds of the static friction force, the statistical summation of asperity contacts corresponding to positive slopes is no longer equal to that for contacts with negative slopes. According to (Bengisu and Akay 1997) the assisting and resisting contacts balance in the case of sliding, with the expected conclusion that friction force is a result of mainly adhesive forces. It is expected that as tangential applied load is increased an d the tangential contact loads due to positive -slope contacts continue to rise while those due to negative -slope contacts decrease. In the limiting case, or the so -called maximum static friction, it is postulated that the load is completely supported by p ositive-slope contacts. The views forwarded by Bengisu and Akay and postulated herein perhaps represent two extremes of what may actually take place in a frictional contact. In the former, the friction is assumed to be mainly due to the adhesive forces whereas in the latter the friction force is assumed to be borne solely by mechanical interaction of roughness. In this way we can obtain a generic formulation that can be easily adapted to any assumption. The scenario for contact of asperities when a maximum force occurs is shown in Fig. 3 . Likewise, $F_{n v}^{-}$and $F_{n v}^{+}$are those forces due to viscous effects, ie. the rate dependent effect of interference at positive and negative asperity slopes. The elastic and viscous terms are a consequence of a viscoelastic contact, but they are defined separately here for the purpose of implementing the approximate analytical description of each term derived later. The tangential force, $F_{t}$, in Eq. (2) exerted through the frictional contact on mass $M$. It is the scalar sum of the elastic interactions of the collection through both resisting and assisting viscoelastic contact components .

$$
F_{t}=F_{t e}^{-}+F_{t e}^{+}+F_{t v}^{-}+F_{t v}^{+}
$$

\subsection{Approximation of Elastic Contact Forces}

The elastic normal contact force per unit area is the sum of the resisting and assisting elastic normal contacts at the asperity interference slopes

$$
F_{n e}=F_{n e}^{-}+F_{n e}^{+}
$$

The analytical solution of the force in Eq. (5) may be approximated as the product of the two terms as follows:

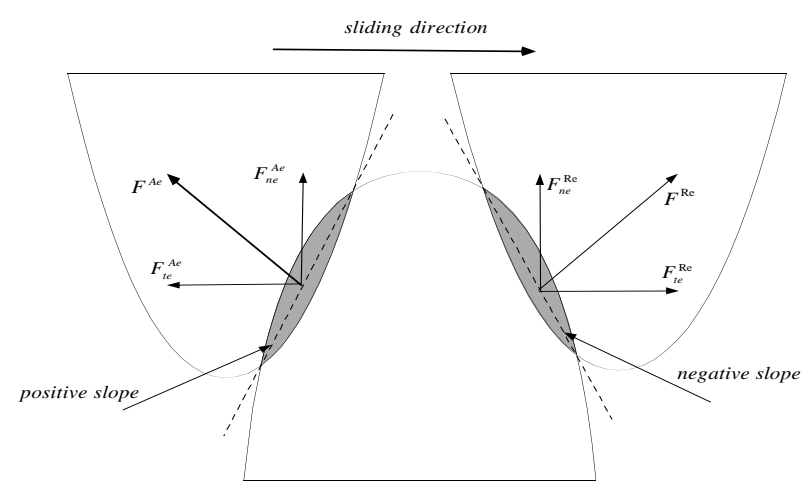

Figure 2. Asperity shoulder contact at positive and negative interference slopes

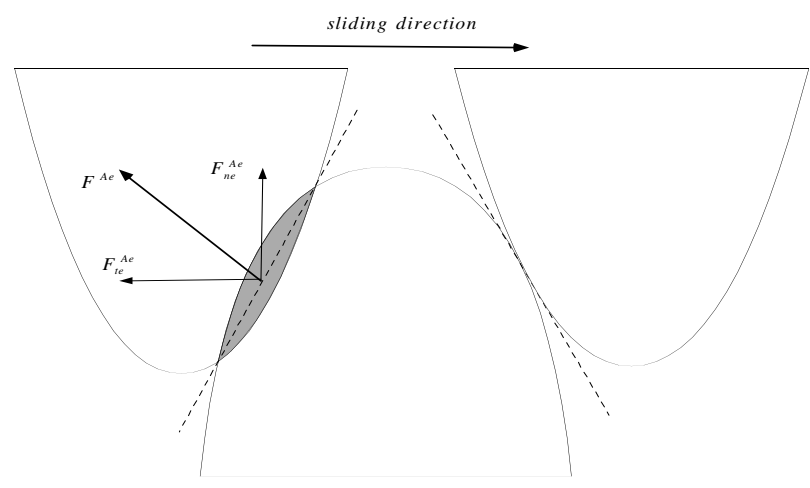

Figure 3. Maximum possible static friction of asperity

$$
F_{n e}=D_{n e} f_{n e}
$$

where $D_{n e}$ and $f_{n e}$, are a function of surface properties and a function of dimensionless mean plan separation between two rough surfaces, respectively. Two integrations are involved in the contact force calculations. The function $D_{n e}$ is defined in Abdo (2006) as

$$
D_{n e}=\frac{8}{3} E^{\prime} c^{2}{ }^{2} \sqrt{0.5 ð} \sqrt{\text { â }}{ }^{4}
$$

$\hat{a}, \quad c ̧, E^{\prime}$, ó are the normalized radius of curvature of asperity mean summit, asperity density, composite modulus of the material and standard deviation of asperity height distribution, respectively. The integral part of the normal force per unit area is

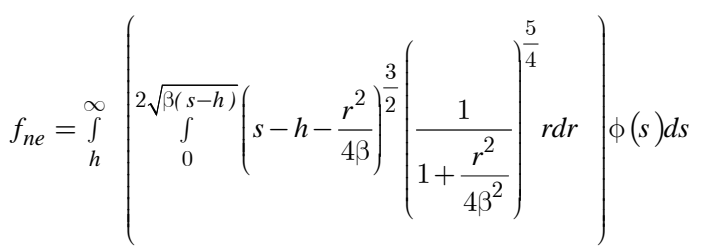

where $h$ is the normalized mean plane separation. The $f_{n e}$ function consists of double integral function over $r$ and $s$ domains. The integration of the contact function occurs 
over the range of asperity-offset misalignment, referred to as $r$-integration. This integration is to account for the total expected interference. The height sum of the rough surface, $s$, is represented by a Gaussian probability density function, $\phi(s)$. Hence, in relating micron-scale events to the resulting macro-scale expectations, two integrations must be performed: firstly, the integration over possible asperity offset and secondly the statistical integration over all possible asperity height sums, s-integration, by implementing a Gaussian probability function. The resulting integral forms describing an elastic contact of rough surfaces are analytically non-integrable. Hence, their solution must be obtained numerically. This fact imposes serious limitations on their use in a dynamic analysis of a mechanical system. To circumvent this shortcoming, we developed approximate analytical functions for viscoelastic contact of rough surfaces. Using an interactive search involving dynamic plotting of the function, $f_{n e}$ is approximated using a considerably simpler function. The result is an approximate function that depends on two independent variables, $\beta, h$. Using the dynamic plotting technique, $f_{n e}$ can be approximated by a product of exponential function of $h$ and a polynomial function of $\beta$. The piecewise analytical solution of $f_{n e}$ is obtained at three different intervals as shown in the following equations:

$$
\begin{gathered}
f_{\text {ne1 }}=\left[\begin{array}{c}
(-0.399+1.8 \beta) h+(-0.389+1.18 \beta)] e^{-3 h} \\
0 \leq h \leq 1.5
\end{array}\right. \\
f_{\text {ne2 }}=\left[\begin{array}{c}
(-1.99+9.538 \beta) h] e^{-3.8 h} \\
1.5 \leq h \leq 3.0
\end{array}\right. \\
f_{\text {ne3 }}=\left[\begin{array}{c}
(-114.96+320.01 \beta) h] e^{-4.9 h} \\
3.0 \leq h \leq 4.5
\end{array}\right.
\end{gathered}
$$

The analytical solution of elastic tangential contact per unit area is developed. The analytical approximation can be described as the product of two functions: a function of surface properties $D_{t e}$ and a function of normalized mean plane separation, $h$, between two rough surfaces, $f_{t e}$, as follows:

$$
F_{t e}=D_{t e} f_{t e}
$$

Note that the constants $D_{n e}$ and $D_{t e}$ are identical. $f_{t e}$ is expressed as

$$
\left.f_{t e}=\int_{h}^{\infty} \int_{0}^{2 \sqrt{\beta(s-h)}}\left(s-h-\frac{r^{2}}{4 \beta}\right)^{\frac{3}{2}}\left(\frac{1}{1+\frac{r^{2}}{4 \beta^{2}}}\right)^{\frac{5}{4}} \frac{r^{2}}{2 \beta} d r\right) \phi(s) d s
$$

Using dynamic fitting technique, the piecewise analytical solution of the function $f_{t e}$ is approximated in closedequations for two intervals of $h$ as

$$
\begin{gathered}
f_{\text {te } 1}=\left[\begin{array}{c}
\left(5.3001+02396 \beta-0.000598 \beta^{2}\right) h \\
+\left(1.694+0.0847 \beta-0.00029 \beta^{2}\right) \\
0 \leq h \leq 2.7
\end{array}\right] e^{-3.5 h} \\
f_{\text {te } 2}=\left[\begin{array}{c}
\left.\left(192.79+9.1601 \beta-0.02498 \beta^{2}\right) h\right] e^{-4.8 h} \\
2.7<h \leq 4.5
\end{array}\right.
\end{gathered}
$$

\subsection{Approximation of Viscoelastic Contact Forces}

The viscoelastic model is developed using the Kelvin-Voigt's constitutive equation relating stress to strain. In Kelvin -Voigt model, the composite modulus of the material $E^{\prime}$ and the loss modulus $E_{v}$ are used to introduce the viscoelastic modulus differential operator

$$
E^{\prime}+E_{v} \frac{\partial}{\partial t} \text {. }
$$

Therefore, a modified form of Hertz contact results in which the modulus operator is used instead of the usual elastic modulus

$$
F=\frac{4}{3} \sqrt{\beta}\left(E^{\prime}+E_{v} \frac{\partial}{\partial t}\right) \omega^{\frac{3}{2}}
$$

where $\omega$ the interference between the two asperities and is derived in (Abdo 2005; and Abdo et al. 2005) as

$$
\omega=\left(s-h-\frac{r^{2}}{2 \beta}\right) \frac{1}{\sqrt{1+\frac{r^{2}}{4 \beta^{2}}}}
$$

Substitution of modulus operator in the Hertz equation of contact and subsequent expansion revealed two forces. One is an elastic force having the form encountered previously, and a second is viscous force defined by

$$
F_{v}=\frac{4}{3} \sqrt{\beta} E_{v} \frac{\partial}{\partial t}\left(\omega^{\frac{3}{2}}\right)
$$

The viscoelastic properties are presented by combining loss modulus with Young's modulus to obtain a differential operator on the interference, reminiscent of the Kelvin-Voigt model. To develop an expression for the normal and tangential components of the viscous forces, the formulations of low to moderate contact force between interacting asperities developed in Abdo (2005) and verified in Abdo (2006) are considered. The interacting asperities are not assumed to occur only at the asperity peaks, thus allowing the possibility of oblique contacts wherein the local contact surfaces are no longer parallel to the mean planes of the mating surfaces. The normal component of the viscous force can be written so as to include the oblique contact 


$$
F_{v}=F_{n v}^{+}+F_{n v}^{-}=\frac{4}{3} \beta^{\frac{1}{2}} E_{v} \frac{1}{\sqrt{1+s_{\alpha}^{2}}} \frac{\partial}{\partial t} \omega^{3 / 2}
$$

where $s_{\alpha}$ is the interference slope defined by two points of interactions with respect to the mean plane. To simplify Eq . (19), we co nsider first the derivation of the rate of change of the interference function, $\dot{\omega}$

$$
\frac{\partial}{\partial t}\left(\omega^{\frac{3}{2}}\right)=\frac{3}{2} \dot{\omega} \omega^{\frac{1}{2}}
$$

Since the interference, $\omega$, depends on the normalized mean plane separation, $h$, and the tangential offset between two asperities, $r$, its derivative with respect to time may be written as

$$
\dot{\omega}=\frac{\partial \omega}{\partial h} \dot{h}+\frac{\partial \omega}{\partial r} \dot{r}
$$

Utilizing Eq. (18) then

$$
\dot{\omega}=-\frac{1}{\sqrt{\frac{4 \beta^{2}+r^{2}}{\beta^{2}}}} \dot{h}-\frac{r}{4 \beta}\left(\left(2+\frac{(s-h)}{\beta}+\frac{r^{2}}{4 \beta^{2}}\right) /\left(1+\frac{r^{2}}{4 \beta^{2}}\right)^{\frac{3}{2}}\right) \dot{r}
$$

The total expected contact force per unit area introduced in the (Greenwood and Trip 1971) is employed to develop the total expected viscous normal contact force per unit area as follows

$$
F_{n v}=2 \pi \eta^{2} \int_{s} \int_{r} F_{n v}^{R e}(\omega, r) \phi(s) r d r d s
$$

Utilizing Eq. (19) through Eq. (22), then Eq. (23) may be written as

$$
\begin{aligned}
F_{n v}= & \frac{4 \pi}{\sqrt{2 \pi}} \eta^{2} \sqrt{\beta} E^{\prime} \eta_{v} \sigma^{4} \int_{h}^{\infty} \int_{0}^{2 \sqrt{\beta(s-h)}} \frac{1}{\left(1+\frac{r^{2}}{4 \beta^{2}}\right)^{3 / 4}} \\
& \left(s-h-\frac{r^{2}}{4 \beta}\right)^{\frac{1}{2}} \dot{\omega} e^{-\frac{s^{2}}{2}} r d r d s
\end{aligned}
$$

where $\eta_{v}=\frac{E_{v}}{E^{\prime}}$ is the loss factor for the friction film or material and $\sigma$ is the standard deviation of the surface heights. The solution for the viscous contact normal force consists of two parts, involving the rate change of the normalized mean plane separation, $\dot{h}$, and the rate of change of the normalized tangential misalignment, ie. the sliding speed, $\dot{r}=\dot{x}-\dot{y}$. The total expected viscous contact nor mal force, due to the interaction on both shoulders, is

$$
\begin{aligned}
& F_{n v}=F_{n v}^{-}+F_{n v}^{+} \\
& \quad=D_{n v}\left(f_{n v h} \dot{h}+f_{n v r} \dot{r}\right)
\end{aligned}
$$

where $D_{n v}$ is a constant term associated with $\dot{h}$ and $\dot{r}$ that depends on the surface parameters and material properties. It is define as follows

$$
D_{n v}=-4 \sqrt{0.5 \pi} \sqrt{\beta} \eta^{2} E^{\prime} \eta_{v} \sigma^{4}
$$

$f_{n v h}$ and $f_{n v r}$ are the analytical functions for the normalized viscous normal force. Using the dynamic plotting technique, the piecewise analytical solutions for $f_{n v h}$ and $f_{n v r}$ can be approximated by a product of exponential function of $h$ and a polynomial function of $\beta$ as follows

$f_{n v h}=\left\{\begin{array}{lc}f_{n v h 1}==((-3.759+5.056 \beta) h+0585+1289 \beta) e^{-3.2 h}, \\ & 0 \leq h \leq 2.7 \\ f_{n v h 2}=(-87.8+209.69 \beta) h e^{-4.5 h} & 2.7 \leq h \leq 45\end{array}\right.$

$$
f_{n v r}=\left\{\begin{array}{l}
f_{n v r 1}=\left(\begin{array}{l}
\left(5.567+02879 \beta-0000804 \beta^{2}\right) \\
h+2.154+0.095 \beta-0.000206 \beta^{2}
\end{array}\right) e^{-3.2 h}, \\
f_{n v r 2}=\left(285.197+1401 \beta-00389 \beta^{2}\right) h e^{-4.6 h}, 27 \leq h \leq 45
\end{array}\right.
$$

In a similar manner, the solution of viscous tangential contact force is developed. The viscoelastic tangential force is

$$
F_{t v}^{-}=F_{t v}^{+}=\frac{4}{3} \beta^{\frac{1}{2}} E_{v} \frac{s_{\alpha}}{\sqrt{1+s_{\alpha}^{2}}} \frac{\partial}{\partial t} \omega^{\frac{3}{2}}
$$

The total expected viscous tangential contact force may be written as

$$
F_{t v}=2 \pi \eta^{2} F_{t v}^{-}(\omega, r) \phi(s) r d r d s
$$

Utilizing Eqs. (20) through (22) and Eq. (29), then Eq. (30) may be written as

$$
\begin{aligned}
F_{t v}=4 \sqrt{0.5 \pi} \eta^{2} A_{t v} \sqrt{\beta} E^{\prime} \eta_{v} \sigma^{4} \int_{h}^{\infty} \int_{0}^{2 \sqrt{\beta(s-h)}} \frac{1}{\left(1+\frac{r^{2}}{4 \beta^{2}}\right)^{3 / 4}} \\
\left(s-h-\frac{r^{2}}{4 \beta}\right)^{\frac{1}{2}}\left(\frac{r}{2 \beta}\right) \dot{\omega} e^{-\frac{s^{2}}{2}} r d r d s
\end{aligned}
$$

As before, the solution for the viscous contact tangential force per unit area consists of two parts involving $\dot{h}$ and $\dot{r}$. Therefore, Eq. (31) may be written as 


$$
F_{t v}=2 D_{t v}\left(f_{t v h} \dot{h}+f_{t v r} \dot{r}\right)
$$

where $D_{t v}$ is identical to $D_{n v}$. $f_{t v h}$ and $f_{t v r}$ are the analytical functions for the normalized viscous tangential force. Using the dynamic plotting technique, the piecewise analytical solutions for $f_{t v h}$ and $f_{t v r}$ can be approximated by a product of exponential function of $h$ and a polynomial function of $\beta$ as follows

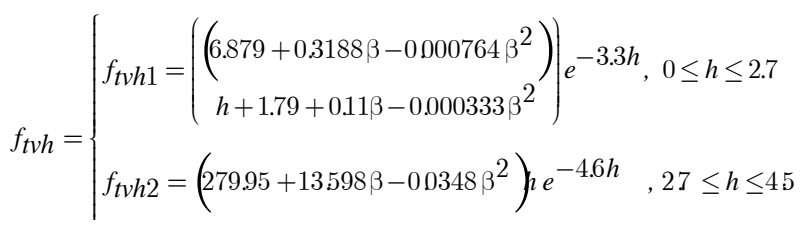

and

$$
f_{\text {tvr }}=\left\{\begin{array}{lr}
f_{\text {tvr } 1}=(0.708+3.195 h) e^{-3.5 h} & 0 \leq h \leq 2.7 \\
f_{\text {tvr } 2}=97.98 h e^{-4.6 h} & 2.7 \leq h \leq 4.5
\end{array}\right.
$$

\subsection{The Model Analysis and Simulation}

According to the dynamic model described in Fig. (1), the dimensionless mean plane separation between two surfaces is

$$
h=h_{o}-\tilde{h}
$$

where $h_{o}$ is the initial mean plane separation of the contacting rough surfaces due to an applied normal force and $\tilde{h}(\hat{h} / \sigma)$ is the dimensionless displacement in the normal direction.

$$
\begin{gathered}
m \ddot{h}=F_{n a}-D_{n e} f_{n e}-D_{n v}\left(f_{n v h} \dot{h}+f_{n v r} \dot{r}\right) \\
m \ddot{x}=-k x-c \dot{x}+D_{t e} f_{t e}+D_{t v}\left(f_{t v h} \dot{h}+f_{t v r} \dot{r}\right)
\end{gathered}
$$

Eqs. (37) and (38) govern the motion of mass $M$ in the normal and tangential directions. The equations tie the vibration behavior of the system to both structural and contact properties of the friction interface.

The dynamic model was developed based on several assumptions to simplify the study. The elastic normal and tangential contact components are due to both positive and negative interference slopes of an asperity as shown in Fig. 2 and are added algebraically to provide their combined effect.

There may or may not be a net resisting elastic tangential force when the two surfaces in relative sliding contact. When this force exists, it is described as a fraction of the maximum static friction force that is due to only positive slopes.

The viscoelastic tangential forces are only produced by the resisting contacts.
When $\dot{h}$ is negative, all viscoelastic components exist and are additive for normal motion. The responses of the dynamic model are studied at different belt speeds and a dimensionless average radius of a summit curvature, $\beta$, to investigate the roughness effect. The equation of motion was solved using a Matlab Simulink program. Unlike in other publications the friction force is accounted for as a result of interaction of the rough surfaces and response of the system. Therefore, the tangential -to-normal contact ratio is a time-dependent variable based on structure and contact characteristics of the system. Table 1 summarizes the parameters used. The simulations include moderate applied load condition corresponding to a high initial separation, $h_{0}=3.5$. The total range of dimensionless mean plane separation is from 0 to 4.5 for contact force functions. The $\beta$ values considered in the simulation are 100 and 140 to ensure low plasticity index so that the asperity deformation will remain predominantly elastic. For cases performed but in order to reduce the length of the article only the results of case 1 will be presented.

Table 1. Parameters from a steel sample

$\begin{array}{llll}\sigma(\mu m) & 15 & \mathrm{~K}_{\mathrm{n}}(\mathrm{N} / \mathrm{m}) & 3.5 \times 10^{8} \\ \eta\left(\mathrm{mm}^{-2}\right) & 400 & \mathrm{C}(\mathrm{N} . \mathrm{s} / \mathrm{m}) & 21166 \\ \eta_{v} & 0.001 & h_{0} & 3.5 \\ E^{\prime}(\mathrm{Gpa}) & 113.1 & \beta & 100 \\ M(\mathrm{Kg}) & 5 & \dot{y}(\mathrm{~m} / \mathrm{s}) & 0.015 \\ \text { Case 1 } & F_{n a}=0.002 F_{c} & & \end{array}$

\section{Results}

The analytical solutions of normal to tangential contact forces presented in this work are applicable to dimensionless mean plane separation, $h=0$ to 4.6 and the dimensionless average radius of asperity summit curvature, $\beta=10$ to 140 . The approximate analytical solutions developed included the normal and tangential contact forces due to viscoelastic effects. The purely elastic term or rate dependent term is presented as a product of two functions. The first corresponds to a constant term for a given surface and the second depends on the separation of mean surfaces as well as tangential asperity offset. It suffices to evaluate the approximate equations with respect to these functions. Therefore the evaluations will only involve $f_{n e}, f_{t e}, f_{n v h}, f_{t v h}, f_{n v r}$ and $f_{t v r}$, not the constant terms $D_{n e}, D_{t e}, D_{n v}$, and $D_{t v}$. The comparison between the original integral function of the elastic normal and tangential contact forces, $f_{\text {ne }}, f_{\text {te }}$ as depicted in Eqs. 8 and 13, respectively, and their piecewise analytical solutions $f_{n e 1}, f_{n e 2}$ and $f_{n e 3}$ and $f_{\text {te } 1}, f_{\text {te } 2}$ and $f_{\text {te } 3}$ for the ranges of $h=0$ to $1.5, h=1.5$ to 3.0 and $h=3.0$ to 4.5 at diff erent values of $\beta$ are performed. The comparisons at $\beta=10,40,75,100,125$, and 140 
show good agreements between the original and the approximation functions throughout the ranges of $\beta$. The relative percentage errors are between $9 \%$ and $10 \%$. The comparisons of the original and viscous functions for the same values of $\beta$ are also performed. The estimation of viscous the functions shows a relative percentage error between the original and the approximation functions is less than 20\%. This is below the inherent uncertainty involved in surface measurements that can climb to as much as $50 \%$. Comparisons between the original integral function of the elastic normal contact force, $f_{n e}$, as depicted in Eq. 8 and its piecewise analytical solutions, $f_{n e 1}, f_{n e 2}$ and $f_{n e 3}$ for the ranges of $h=0$ to $1.5, h=1.5$ to 3.0 and $h=3.0$ to 4.5 , respectively, at $\beta=100$ and 140 are shown in Fig. 4. As seen in the figures, the comparisons show good agreement between the original and the approximate functions for $\beta=100$ and 140 . The relative percentage error between $f_{n e}$ and $f_{n e 1}, f_{n e 2}$ and $f_{n e 3}$ is $9 \%$ at $\beta=100$ and $10 \%$ at $\beta=140$. The comparison between the approximate functions $f_{t e}$ and its analytical solutions and $f_{t v h}$ and its analytical solutions for the ranges of $h=0$ to 1.5 , $h=1.5$ to 3.0 and $h=3.0$ to 4.5 , respectively, at $\beta=$ 100 and 140 are shown in figures 5 and 6 . The relative percentage error between the original and the approximation functions for both functions are $17 \%$ and $19 \%$, respectively.

The results for case (1) are depicted in Fig. 7 through Fig. 10. Figure 7a shows the trajectory of tangential motion, tangential speed versus tangential displacement of the mass $m$. The zoom-in view (Fig. 7b) shows decreasing spiral, which represents a mass response of decaying oscillation corresponding to a stable behavior. The trajectories in Fig. 7 and the time history in Fig. 8 indicate an initial decay followed by a growth in vibration up to a level at which the vibration is sustained.

In case of the normal motion of the mass the structural decay is absent consistent with the system's depiction in Fig. 1 in which no structural stiffness or damping exists for the vibration of mass $M$ along the normal to the platform. As shown in Fig. 9(a,b) the vibration is grown and sustained at a value of $0.005 \mathrm{~mm}$. The friction coefficient defined as the tangential to normal friction shown in Fig. 10 depicts a steady state value of 0.026 for case 1 .

\section{Conclusions}

This paper has addressed the dynamic effects of the surface irregularities (roughness) as a result of mechanical interactions. We have exhibited the cross-influence of tribological interactions that occur at the micron-scale and the dynamics of a mechanical system involving macroscale events. The utility of the contact theories in this work was demonstrated through the dynamic analysis of a simple mechanical system. We have considered a mechanical system comprising a spring-damper-mass and a platform in relative tangential and normal movements while maintaining frictional contact between mass and platform rough surfaces.

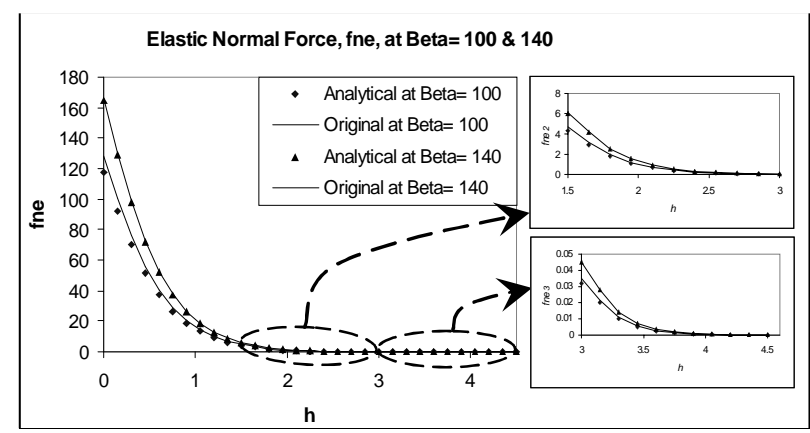

Figure 4. Elastic normal force at $\beta=100 \& 140$

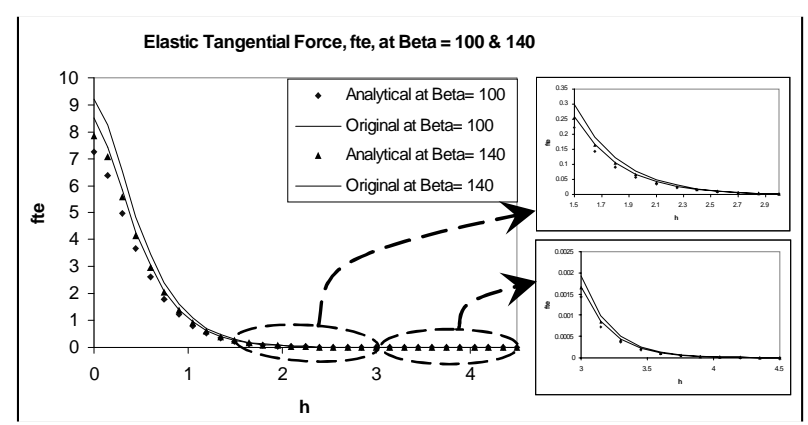

Figure 5. Elastic tangential force at $\beta=100 \& 140$

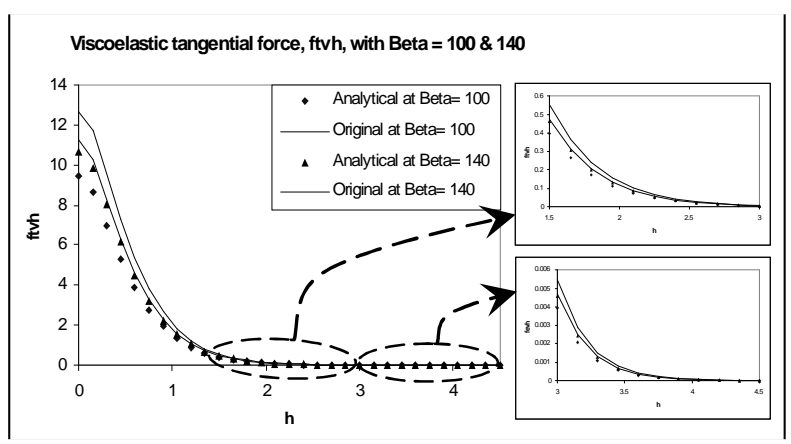

Figure 6. Viscoelastic tangental force at $\beta=100$ \& 140

The results pertaining to the prediction of contact forces were used along with the addition of the viscoelastic effects of contact. The approximate analytical solutions were obtained for normal and tangential contact components in viscoelastic contact. The term viscoelastic was separated into components of elastic and rate-dependent interactions. It was shown that the formulation of contact forces allow the inclusion of frictional contact without the necessity of including the friction/velocity relation phenomenologically. No presumption of a friction coefficient has been made. The friction force is accounted for as a result of interaction of the rough surfaces. Rather the tangential (friction) force has been a result of the consideration of contact at the micron-scale level between two asperities on the surfaces and the statistical summation of this interaction to obtain the macrolevel expectation functions. This led to a set of nonlinear ordinary differential 


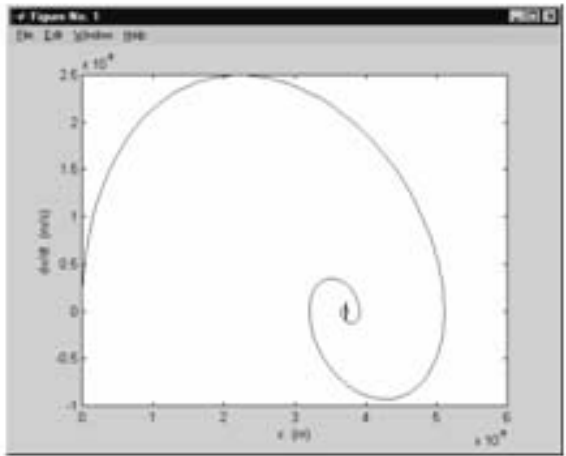

(a)

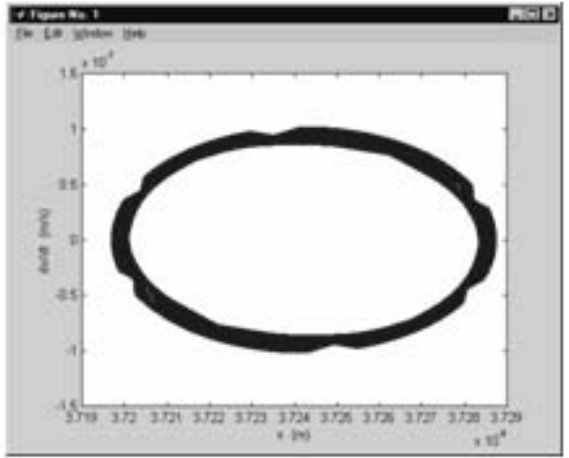

(b)

Figure 7 (a, b). Trajectory of tangential motion and the zoom-in view, Case 1

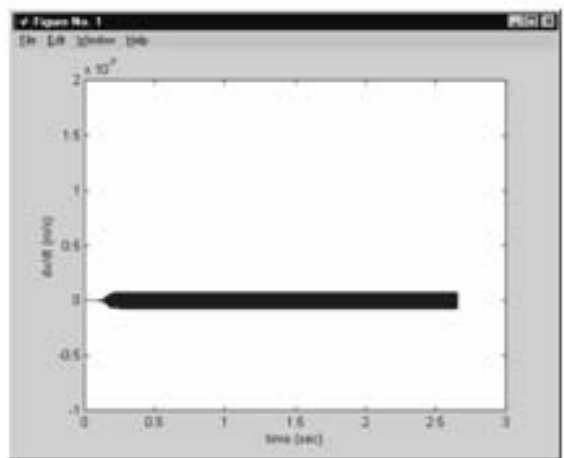

(a)

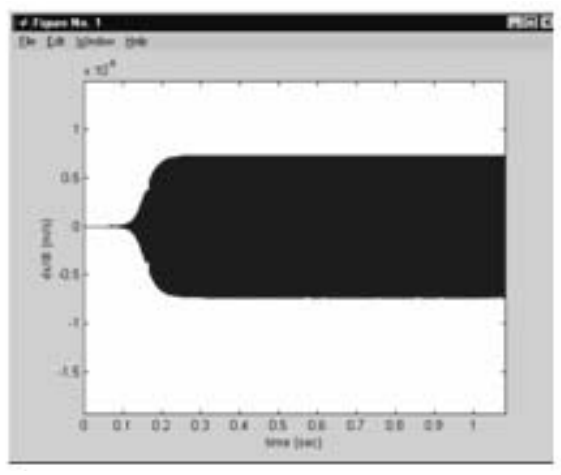

(b)

Figure $8(a, b)$. Tangential speed of the mass and the zoom-in view, Case 1

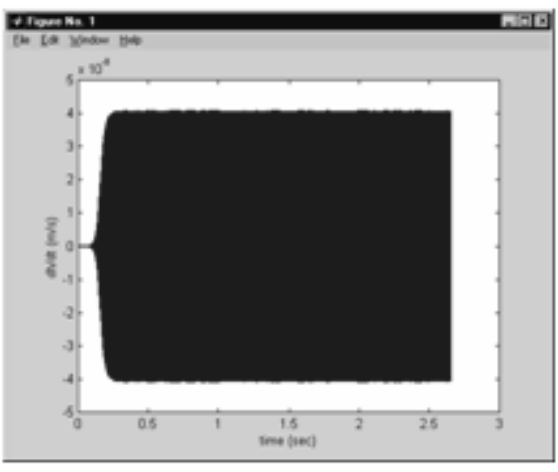

(a)

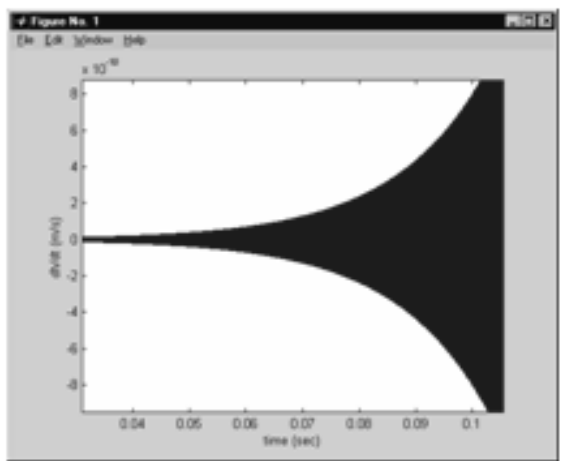

(b)

Figure 9 (a, b). Normal speed of the mass and the zoom-in view, Case 1

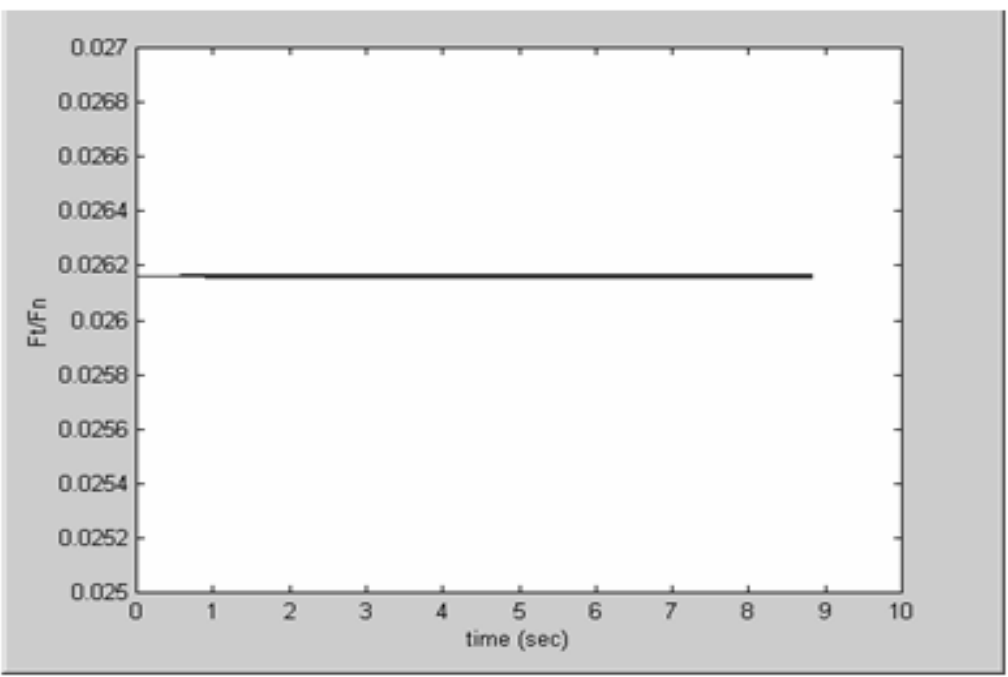

Figure 10 (a, b). Load ratio (friction function), Case 1 
equations that directly relate the vibration of the system to the surface parameters, mechanical system parameters and physical parameters.

The study has shown the following:

- The tangential trajectories of the four cases showed initial decay dominated by structural damping followed by a growth in vibration up to a level at which vibration is sustained that is due to the interaction of surface asperities of the moving platform and those of the structure mass.

- The vibration of the normal direction of the four cases grow to a level at which it is sustained that is due to frictional interaction between the platform surface roughness and that of mass $M$ and the absence of the structural decay.

- The low tangential to normal contact load ratio of the four cases is due in part to the relatively low surface roughness in the present study in order to ensure the predominance of elastic interaction at the contact.

\section{References}

Abdo, J. A. and Farhang, K., 2005, "Elastic-Plastic Contact Model for Rough Surfaces based on Plastic Asperity Concept," Int. J. of Non-Linear Mechanics, Vol. 40, pp 495-506.

Abdo, J. A. and Shamseldin, E., 2005, "Modeling of Contact Area, Contact Force, and Contact Stiffness of Mechanical Systems with Friction," ASME Mechanical Engineering Congress Paper no. IMECE2005-82980, Orlando, Florida.

Abdo, J. A., 2005, "Experimental Technique to Study Tangential-to-normal Contact Load Ratio," Tribology Transactions, Vol. 48, pp 389- 403.

Abdo, J. A., 2006, "Modeling of Normal and Tangential Contact Stiffness of Rough Surfaces," Int. J. of Modeling and Simulation, Vol. 26(4), pp 295-302.

Aronov, V., D'souza, A. F., Kalpakjian, S. and Sharper, I., 1984a, "Interaction among Friction, Wear and System Stiffness-Part 1: Effect of Normal Load on System Stiffness," J. Tribol., Vol. 106, pp. 54-58.

Aronov, V., D'souza, A. F., Kalpakjian, S. and Sharper, I., 1984b "Interaction among Friction, Wear and System Stiffness-Part 2: Vibrations Induced by dry Friction," J. Tribology, V. 106, pp. 59-64.

Aronov, V., D'souza, A. F., Kalpakjian, S. and Sharper, I., 1984c "Interaction among Friction, Wear and System Stiffness-Part 3: Wear Model," J. Tribology, Vol. 106, pp. 59-64.

Bengisu, M. T. and Akay, A., 1997, "Relation of Dry Friction to Surface Roughness," ASME J. of Trib, Vol. 119, pp. 18-25.
Bengisu, M. T. and Akay, A., 1999, "Stick-Slip Oscillations: Dynamics of Friction and Surface Roughness," J. of Acoust. Soc. Am., Vol. 105, pp. 194-205.

Brockley, C. A. and Ko, P. L., 1970, "The Measurement of Friction and Friction-Induced Vibration - Trans. ASME - pp. 543-549.

Greenwood, J. A. and Tripp, J. H., 1971, "The Contact of Two Rough Nominally Flat Rough Surfaces," Proc. Instn. Mech. Engrs., Vol. 185, pp. 625-633.

Ibrahim, R. A., 1994, "Friction Induced Vibration, Chatter, Squeal and Chaos, Part II: Dynamic and Modeling - Applied Mechanics Reviews," Vol. 47, pp. 227-253.

Ibrahim, R. A. and Rivin, E., 1994, "Friction-Induced Vibration, Part I: Mechanics of Contact and Friction Applied Mechanics Reviews," Vol. 47, pp. 209-226.

McMillan, J., 1997, "A Non-linear Model for Self-excited Vibration," J. Sound Vibration, Vol. 205(3), pp 323335.

Mitropolskii, Y.A. and Nguyen, V. D., 1997, "Applied Sympotic Methods in Nonlinear Oscillation," Kluwer, Dordrecht.

Nayfeh, A. H. and Mook, D. T., 1979, "Nonlinear Oscillations," Wiley, New York.

Panovko, Y. G. and Gubanova, I. I., 1965, "Stability and Oscillation of Elastic Systems; Paradoxes, Fallacies and New Concepts," Consultants Burea, New York.

Popp, K., 1992, "Some Model Problems Showing Stickslip Motion and Chaos, Friction-Induced Vibration, Chatter, Squeal, and Chaos," ASME DE, Vol. 49, ASME Design Engineering Division, New York, pp. 1-12.

Soom, A. and Kim, C., 1983, "Interactions between Dynamic Normal and Frictional Forces during Unlubricated sliding," Transactions of the ASME, J. of Lubrication Technology, Vol. 105, pp. 221-229.

Tan, X. and Roger, R., 1998, "Simulation of Friction in Multi-Degree-of-Freedom Vibration System," ASME J. Dyn. Syst., Control, Vol. 120, pp. 144-146.

Thomsen, J. J. and Fidlin, A., 2002, "Analytical Approximations for Stick-slip Vibration Amplitudes," Int. J. of Non-Linear Mechanics, Vol. 38, pp 389-403.

Tondl, A., 1991, "Quenching of Self-Excited Vibration," Elsevier, Amsterdam.

Tworzydlo, W., Becker, E. and Oden, J., 1992, "Numerical Modeling of Friction-Induced Vibration and Dynamic Instabilities," App.l Mech. Rev. Vol. 45(7), pp. 255274.

Tzou, K., Wickert, J. and Akay, A., 1998, "In-plane Vibration Modes of Arbitrarily Thick Disks," ASME J. Vibr. Acout., Vol. 120, pp. 384-391. 